\title{
The Efficacy and Safety of Bosutinib in Chronic Myeloid Leukemia
}

\author{
Nahla AM Hamed* \\ Department of Hematology, Alexandria University, Egypt
}

Submission: April 23, 2018; Published: April 30, 2018

"Correspondence Address: Nahla AM Hamed, Professor of Hematology, Faculty of Medicine, Alexandria University, Egypt, Email: drhamedn@hotmail.com

Abstract

Dasatinib and nilotinib are second-generation TKIs that demonstrated efficacy in CP-CML patients resistant or intolerant to imatinib, with 53\%-59\% of patients achieving MCyR. Unfortunately, resistance or intolerance to dasatinib and/or nilotinib treatment also develops in some patients. Few treatment options remain for patients who are previously treated with imatinib and dasatinib and/or nilotinib. Alternative treatments options are needed for CP-CML patients after treatment failure and resistance or intolerance to multiple TKIs.

Abbreviations: CML: Chronic Myeloid Leukemia; SRC/ABL: Src and Abelson; CP: Chronic Phase; AP: Accelerated Phase; BP: Blast Phase; VEGFR: Vascular Endothelial Growth Factor Receptor; MCyR: Major Cytogenetic Response; TEAEs: Treatment-Emergent Adverse Events; PPI: Proton Pump Inhibitors; IM-I: Imatinib Intolerant; IM-R: Imatinib Resistant; TKI: Tyrosine Kinase Inhibitor

\section{Introduction}

Bosutinib is a second-generation TKIs with dual inhibition of Src/Abl kinases [1], and minimal inhibitory activity against c-KIT or platelet-derived growth factor receptor [2]. It is currently approved in Europe and USA for treatment of CP, AP, and BP Philadelphia chromosome-positive adult CML patients previously treated with one or more TKI and also for whom imatinib, nilotinib, and dasatinib are inappropriate treatment options [1].

Bosutinib is effective in most imatinib-resistant mutations except T315I and V299L [3]. Bosutinib also demonstrated confirmed CHR (50\%-83\%) and MCyR (14\%-67\%) in baseline mutations associated with clinical resistance to dasatinib (F317L) and nilotinib (Y253H and F359C/I/V) [2]. Bosutinib exposure and time on treatment were significant predictors of MMR, with time on treatment appeared to play a larger role in efficacy [4].

\section{Pharmacokinetic Data}

Bosutinib is primarily metabolized by cytochrome P-450 isoenzyme 3A4; therefore concomitant use of strong or moderate CYP3A4 inhibitors and inducers should be avoided [3]. Concomitant use of PPI is not recommended in patients receiving bosutinib. The use of short-acting antacids or $\mathrm{H} 2$ blocker should be considered instead of PPI [5]. Drug absorption is relatively slow, with a median time to peak concentration of 4-6hr and a half-life t1/2 ranging from 33 to $39 \mathrm{hr}$, supporting a oncedaily dosing regimen [1]. In chronic phase, bosutinib should be administered at a dose of $400 \mathrm{mg} /$ day start and increase the dose to $500 \mathrm{mg}$ /day if tolerated [6]. Bosutinib $500 \mathrm{mg}$ daily is approved as second- or subsequent-line therapy for CML [7].

Specific recommendations concerning dose adjustment were made for severe ( $\mathrm{CLcr}<30 \mathrm{~mL} / \mathrm{min}$ ) or moderate (CLcr between 30 and $50 \mathrm{~mL} / \mathrm{min}$ ) renal impairment. Use of bosutinib is contraindicated in Europe for patients with hepatic impairment [1]. Although there are no data regarding the outcome of pregnancy in patients receiving bosutinib at time of conception, this agent must be considered unsafe to use in pregnant women. Bosutinib has not been tested in children in clinical trials; therefore, there is little information on the safety of this drug in this population [5].

\section{The Efficacy and Safety of Bosutinib as Initial Therapy for CP-CML Patients}

Bosutinib 400mg once daily may be an effective first-line treatment for CP- CML. Patients who received bosutinib had significantly higher MMR and CCyR rates and achieved earlier responses than those who received imatinib [7]. Most AEs are low grade, manageable, and improving over time. The most common AEs are grade 1 or 2 diarrhea and liver function abnormalities. Diarrhea occurred early and was transient and manageable, its frequency and severity typically improve over time. Diarrhea did not lead to a higher discontinuation rate compared with the imatinib arm. The most common AEs leading to bosutinib discontinuation were liver function abnormalities [7]. These results suggest that bosutinib provides benefit over 


\section{Cancer Therapy \& Oncology International Journal}

imatinib for previously untreated CP-CML patients and can be an important alternative [7]. The superiority of bosutinib compared with imatinib for de novo CP-CML could offer another possibility for inducing deep molecular response, particularly for nilotinib and dasatinib therapy ineligible patients [6].

The Efficacy and Safety of Bosutinib after Treatment with Multiple TKIs (Imatinib and Dasatinib and/or Nilotinib)

Bosutinib demonstrated clinical activity and an acceptable safety profile in patients with limited treatment options [2]. Second-line bosutinib therapy after IM-R or IM-I in CP-CML patients generally demonstrated durable clinical activity and manageable toxicity comparable with the reported results for second-line dasatinib and nilotinib therapy [8]. Bosutinib also demonstrated durability of clinical outcomes among CP-CML patients who had previously received imatinib plus dasatinib and/or nilotinib. The MCyR rate with bosutinib is similar, yet with longer median duration, compared with historical results of smaller studies of third-line dasatinib or nilotinib therapies [2]. Responses have also been observed in AP and BP-CML. Bosutinib is also being evaluated in patients with previously treated AP or BP-CML [8].

Treatment-emergent drug-related AEs were primarily manageable grade $1 / 2$ gastrointestinal events (ie, diarrhea, nausea, vomiting) and rash, and were consistent with the AEs reported in patients treated only with prior imatinib. The rate of transaminase elevations was low, with on-treatment grade $3 / 4$ ALT and AST elevations detected in $7 \%$ and $3 \%$ of patients, respectively. Transaminase elevations rarely led to treatment discontinuation ( $3 \%$ and $1 \%$ of patients respectively). The incidence of on-therapy grade 3/4 hypermagnesemia appeared high (12\%) in Khoury et al. [2] analysis.

The incidence of AEs appeared to correlate with bosutinib exposure. Staying on treatment may be more important than receiving a higher dose of bosutinib. Comparing the 2 starting doses of bosutinib suggests that the lower dose of bosutinib used in the BFORE trial $(400 \mathrm{mg} /$ day) did not compromise efficacy but decreased the probability of permanent drug discontinuation due to AEs. Higher bosutinib exposure was associated with a greater probability of diarrhea, nausea and vomiting earlier on treatment, and of rash and elevated ALT and AST at any time on treatment [4].

\section{Cross-Intolerance}

Cross-intolerance of bosutinib used as second or third line treatment after previous TKI therapy was infrequent. Bosutinib was discontinued in $8 \%$ of patients because of cross-intolerance with dasatinib (the same $\mathrm{AE}$ attributed to dasatinib intolerance). Grade 3/4 hematologic cross-intolerance event on bosutinib was experienced in $8(40 \%)$ and myelosuppression necessitating bosutinib discontinuation occurs in $2(10 \%)$ in Khoury, et al. studied patients. However, the overall incidence of on-therapy grade $3 / 4$ neutropenia (19\%) and thrombocytopenia (25\%) was relatively low. These incidences were comparably favorable with the reported events rates in the literature for dasatinib and were similar to rates reported with second-line nilotinib therapy [2].

\section{Vascular and Cardiac TEAEs}

Bosutinib treatment was notable for a low incidence of certain AEs such as pleural effusions, musculoskeletal events and cardiac toxicity that are common with other TKIs [2]. The incidence of vascular (including cerebrovascular, cardiovascular, and peripheral vascular) and cardiac TEAEs during long-term bosutinib therapy is relatively low in the first-line (phase 3 study) and second-/ third-/fourth-line (phase 1/2 study) settings [9]. Cardiac and vascular toxicities were uncommon when comparing bosutinib versus imatinib in newly diagnosed CML patients. In the BFORE study, cardiovascular events occurred in $3.0 \%$ of patients receiving bosutinib and $0.4 \%$ of patients receiving imatinib; $2.2 \%$ and $0.4 \%$, respectively, occurred during the first year of therapy [7]. The incidence of cardiovascular events at 12 months in BELA was 0.8\% in both arms; however, more patients enrolled in the BFORE study had a history of cardiac disorders and vascular disorders [7].

The incidence of heart failure was similar for first-line bosutinib and imatinib (0.8\% vs. $0.8 \%$ ) and low with bosutinib overall (3.3\%) [9]. Based on the standardized MedDRA query, Torsade de pointes/QT prolongation, occurred in only $1.5 \%$ of all bosutinib-treated patients. This rate may be an underestimation, because ECG analyses were not prospectively planned to assess all potential cardiac events [9]. The incidence of vascular emergent side effects with bosutinib was comparable with imatinib in the BELA study [1]. Hypertension, was common with bosutinib (in a combined analysis any grade $\backslash 7 \%$; grade $3 / 4 \backslash 2 \%$ ) [1].

One reason for the distinct cardiac and vascular toxicity profile of bosutinib versus other TKIs may be related to the divergent specificities for BCR-ABL1 versus other important kinases and the more selective off-target effects than other TKIs. In contrast to all other TKIs, bosutinib exhibits minimal inhibitory activity against c-Kit and platelet-derived growth factor receptor [9]. Vascular dysfunction associated with multikinase TKIs, such as ponatinib, is thought to relate to strong inhibition of VEGFR signaling, a pathway affected less by bosutinib than other TKIs [9]. Analysis of the predisposing factors to cardiac events showed that age $>65$ years, previous history of cardiac disorders, ECOG $>0$ in the phase $1 / 2$ study and history of hypertension in phase 3 trial, or hypercholesterolemia (in both studies) were significantly associated [1].

\section{On-Treatment Transformation to Accelerated or Blast Phase}

On-treatment transformation to accelerated phase or blast phase occurred in IM-R (7\%) and IM-I (2\%) patients with no new events in a 5-yr follow-up. The cumulative incidence of on- 
treatment progression or death at 8yr was 28\% (IM-R) and $11 \%$ (IM-I), respectively. Overall survival at $8 y$ r on study was $75 \%$ for the IM-R group and $87 \%$ for the IM-I group [10]. In another study, only $5(4 \%)$ patients experienced confirmed transformation to AP-CML during treatment, and no transformations to BP-CML occurred [2].

\section{Immunological Monitoring of CML patients}

No major changes in T- or NK-cell phenotype or function were observed during first-line bosutinib treatment despite the Src-kinase inhibitory profile of bosutinib. In contrast, the proportion of NK-cells increased and their degranulation responses were significantly higher in imatinib treated patients than in untreated CML patients. Comparison of these data with treatment outcome is warranted [11].

\section{Who are the Best Candidates for Bosutinib?}

Bosutinib could be considered among the treatment options for patients with cardiac or vascular co morbidities [9]. Vascular and cardiac TEAE incidences in leukemia patients receiving bosutinib are generally low, even after long-term treatment relative to other new-generation TKIs, and not significantly different from those observed in imatinib-treated patients. Dose adjustments and discontinuations due to these events were rare [9]. Bosutinib can be added as a treatment option for any risk score (category 1 recommendation). Preliminary data from the BFORE trial suggest that patients with an intermediate- or high risk sokal or hasford score may preferentially benefit from bosutinib [5]. Moreover, bosutinib is active against many BCRABL kinase domain mutations resistant to imatinib, dasatinib, and nilotinib, with the exception of T315I andV299L [1].

\section{References}

1. Breccia M, Binotto G (2015) Bosutinib for chronic myeloid leukemia. Rare Cancers Ther 3: 35-46.

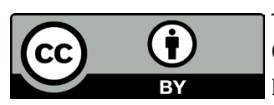

This work is licensed under Creative Commons Attribution 4.0 License

DOI: $10.19080 /$ CTOIJ.2017.10.555787
2. Khoury HJ, Cortes JE, Kantarjian HM, Gambacorti-Passerini C, Baccarani M, et al. (2012) Bosutinib is active in chronic phase chronic myeloid leukemia after imatinib and dasatinib and/or nilotinib therapy failure. Blood 119(15): 3403-3412.

3. Doan V, Wang A, Prescott H (2015) Bosutinib for the treatment of chronic myeloid leukemia. American Journal of Health-System Pharmacy 72(6): 439-447.

4. Mauro MJ, Gambacorti-Passerini C, Deininger MW, Chuah C, Kim D-W, et al. (2017) Bosutinib in newly diagnosed chronic myeloid leukemia (CML): gastrointestinal, liver, and hematological safety characterization in the Bfore trial. Blood 130: 1620.

5. NCCN (2018) NCCN Clinical Practical Guidelines in Oncology (NCCN Guidelines). Chronic myeloid leukemia. Version 4, USA.

6. Mahon FX (2017) Treatment-free remission in CML: who, how, and why? Hematology Am Soc Hematol Educ Program 2017(1): 102-109.

7. Cortes JE, Gambacorti-Passerini C, Deininger MW, Mauro MJ, Chuah C, et al. (2018) Bosutinib versus imatinib for newly diagnosed chronic myeloid leukemia: results from the randomized BFORE trial. J Clin Oncol 36(3): 231-237.

8. Gambacorti-Passerini C, Br€ummendorf TH, Kim DW, Turkina AG, Masszi T, et al. (2014) Bosutinib efficacy and safety in chronic phase chronic myeloid leukemia after imatinib resistance or intolerance: Minimum 24-month follow-up. Am J Hematol 89(7): 732-742.

9. Cortes JE, Khoury HJ, Kantarjian H, Brümmendorf TH, Mauro MJ, et al. (2016) Long-term evaluation of cardiac and vascular toxicity in patients with Philadelphia chromosome-positive leukemias treated with bosutinib. Am J Hematol 91(6): 606-616.

10. Brummendorf TH, Gambacorti-Passerini C, Kim D-W, Goh YT, Dyagil I, et al. (2017) Second-line bosutinib in patients with chronic phase chronic myeloid leukemia (CP-CML) resistant or intolerant to prior imatinib: an 8-year update. Blood 130: 900.

11. Kreutzman A, Koshenvesa P, Tiina K, Olsson-Stromberg U, Stentoft J, et al. (2016) Immunological monitoring of CML patients during first-line bosutinib and imatinib treatment. Blood 128: 3069.

\section{Your next submission with Juniper Publishers will reach you the below assets}

- Quality Editorial service

- Swift Peer Review

- Reprints availability

- E-prints Service

- Manuscript Podcast for convenient understanding

- Global attainment for your research

- Manuscript accessibility in different formats

( Pdf, E-pub, Full Text, Audio)

- Unceasing customer service

Track the below URL for one-step submission https://juniperpublishers.com/online-submission.php 